Gut, 1977, 18, 7-15

\title{
Speed of change in biliary lipids and bile acids with chenodeoxycholic acid-is intermittent therapy feasible? ${ }^{1}$
}

\author{
J. H. ISER, G. M. MURPHY, AND R. HERMON DOWLING ${ }^{2}$ \\ From the Gastroenterology Unit, Department of Medicine, Guy's Hospital and Medical School, London
}

SUMMARY To see whether intermittent chenodeoxycholic acid (CDCA) therapy is a potential alternative to continous treatment for gallstone dissolution, the speed of change in bile lipid composition was studied after starting and stopping CDCA therapy. In addition, the relationship between bile lipid composition and the proportions of the bile acids was examined. Bile-rich duodenal fluid was collected twice in the first week and then at approximately weekly intervals for four to six weeks, from six gallstone patients starting 13-15 mg CDCA.kg BW-1 day ${ }^{-1}$ and from another group of six patients whose treatment was stopped after gallstone dissolution. After starting treatment, the mean biliary cholesterol saturation index (based on criteria of Hegardt and Dam, 1971) decreased from $1.49 \pm$ SEM 0.17 to $0.92 \pm 0.13$ at three weeks and $0.88 \pm 0.10$ at four weeks, by which time bile lipid composition had become relatively constant. In patients whose treatment was stopped, bile reverted to its supersaturated state within one week, changing from an on-treatment mean saturation index of $0.74 \pm 0.10$ to $1.15 \pm 0.15$ in six to eight days after withdrawing CDCA. The proportion of conjugated CDCA in the biliary bile acids increased from $27.9 \pm 2.5 \%$ to $60.5 \pm 4.2 \%$ within four days and to $80.7 \pm 6.2 \%$ by four weeks after starting CDCA. When treatment was stopped, the proportion of CDCA reverted to pretreatment levels by two to three weeks. The saturation index was significantly related $(P<0.001)$ to the percent of conjugated CDCA present, such that when the proportion of CDCA exceeded $70 \%$, bile was almost invariably unsaturated. Since the mean time taken for bile to become unsaturated was not shorter than the time taken for bile to revert to its supersaturated state, it seems that intermittent treatment would not be adequate to maintain an unsaturated bile and is, therefore, unlikely to be as effective as continuous treatment in dissolving gallstones.

It is now known that, with adequate doses, oral chenodeoxycholic acid (CDCA) will dissolve most radiolucent gallstones (Bell et al., 1972a; Danzinger et al., 1972; Hofmann and Paumgartner, 1975).

\footnotetext{
1Presented in part at the autumn meeting of the British Society of Gastroenterology, Oxford, 1975 (Iser, J. H., Murphy, G. M., Dowling, R. H. Is intermittent chenodeoxycholic acid therapy feasible for dissolving gallstones? The speed of change in biliary lipids and bile acids after starting and stopping treatment. Gut, 16, 840 (Abstract)) and at the Second NATO Advanced Study Institute on the Biliary System, Aalborg, 1975 (Dowling, R. H., Murphy, G. M., Iser, J. H. Dissolution of gallstones by chenodeoxycholic acid. In The Hepatobiliary System, pp. 485-501. Ed. W. Taylor, Plenum Publishing Co.)

'Address for reprint requests: Professor R. H. Dowling, Gastroenterology Unit, Guy's Hospital, London Bridge, SE1.
}

Received for publication 6 October 1976
CDCA acts by reducing the secretion of cholesterol into bile (Adler et al., 1975; Northfield et al., 1975) and we have recently shown that, before gallstones can dissolve, the bile must become unsaturated in cholesterol (Iser et al., 1975). To avoid prolonged treatment with ineffective doses of CDCA, therefore, we believe that the bile lipid response to treatment should be monitored in all patients. However, it is not known how soon after starting CDCA the bile becomes unsaturated with cholesterol, nor when one can expect to find representative changes in bile lipid composition.

Furthermore, when CDCA is stopped, the bile reverts to its supersaturated state (Mok et al., 1974a; Thistle et al., 1974) but again it is now known how quickly this happens. This reversion to the supersaturated state is not synonymous with the re-forma- 
tion of gallstones, but in a few patients, whose treatment was stopped when the gallstones dissolved, the stones have recurred (Thistle et al., 1974; Iser et al., 1975). The frequency with which this happens is unknown, but, to prevent it, long-term treatment may be necessary and although there has been no evidence of short-term toxicity (in patients treated for up to four years) (Hofmann and Paumgartner, 1975), the long-term side-effects of CDCA therapy are unknown. To minimise such possible risks and for reasons of economy, intermittent treatment-for example, treatment on alternate months-would seem preferable to continuous long-term therapy, but the efficacy of intermittent CDCA, both in changing bile lipid composition and in dissolving gallstones, is unknown.

The aims of this study, therefore, were to examine the time course of changes in bile lipid composition after starting and stopping CDCA, and, based on these results, to see if intermittent treatment would effectively maintain an unsaturated bile. We also looked at the proportions of the major biliary bile acids to see if the saturation of bile with cholesterol was related to the proportion of CDCA or of the other bile acids in bile.

\section{Methods}

Bile lipid and biliary bile acid composition were measured at frequent intervals after starting and stopping CDCA therapy in two separate groups of patients.

\section{STARTING CDCA TREATMENT}

Six patients (five women and one man) with radiolucent gallstones in functioning gallbladders were studied. Their mean age was 56.7 ( \pm SEM6.6; range 29-78) and their mean body weight was $69.2 \mathrm{~kg}$ ( $\pm 5 \cdot 1$; range $55-95)$.

After an overnight fast, the duodenum was intubated under fluoroscopic control and bile-rich fluid collected after intravenous cholecystokinin (1 unit per $\mathrm{kg}$ body weight of CCK-PZ, G. I. H. Laboratory, Karolinska Institute, Stockholm, Sweden). Bile samples were obtained at day 0 (pretreatment baseline) and subsequently on days $2-4$, 6-8, 12-17, 18-24 and 26-28, and in three patients also on days 38-47 after starting treatment. At each intubation, $4 \mathrm{ml}$ of 'bile' was retained for analysis, the remainder being returned to the duodenum. Samples were stored immediately at $-20^{\circ} \mathrm{C}$ until analysed.

To minimise the variable of different doses of CDCA, a factor known to influence bile lipid composition (Mok et al., 1974a), the patients were given 13-15 mg CDCA. $\mathrm{kg} \mathrm{BW}^{-1} \mathrm{day}^{-1}$, a dose which we have previously shown results in both an unsaturated bile and gallstone dissolution (Iser et al., 1975). The last dose of CDCA was taken with the evening meal on the day before the duodenal intubation, so that the bile was sampled 13-17 hours after the last CDCA capsule.

\section{STOPPING CDCA TREATMENT}

Six women (age 55.8 years \pm 6.8 ; range 28-74, weighing $66 \cdot 7 \mathrm{~kg} \pm 3 \cdot 5$; range $57-80 \mathrm{~kg}$ ) who also had had radiolucent gallstones in functioning gallbladders and whose gallstones had dissolved after an average of 19 months' (range 15-26) treatment with a mean dose of $14.2 \mathrm{mg} \mathrm{CDCA} \mathrm{kg} \mathrm{BW}{ }^{-1}$ day $^{-1}$ (range 11.4-16.7) were studied when CDCA was withdrawn. Bile-rich duodenal fluid was again aspirated just before treatment stopped (day 0 ) and on days 3-4, 6-8, 14-18, 20-22, and 28-31 after stopping therapy.

\section{ANALYSIS OF 'BILE' SAMPLES}

\section{Bile lipids}

All samples were analysed for total bile acids, phospholipids, and cholesterol as previously described (Mok et al., 1974b). The degree of saturation of bile with cholesterol was then calculated as the saturation (lithogenic) index (SI) (Thomas and Hofmann, 1973) where an index of $>1$ is supersaturated and $<1$ undersaturated, according to the limits of cholesterol solubility as defined by Hegardt and Dam (1971).

\section{Biliary bile acids}

To measure the proportion of the individual bile acids in bile, methanolic extracts of the bile samples were separated into the mono- (lithocholic acid), di- 1 (chenodeoxycholic and deoxycholic acids) and trihydroxy- (cholic acid) bile acid bands by thin layer chromatography, using $\mathbf{0 . 2 5} \mathrm{mm}$ silica gel G (Merck, Darmstadt) plates and the solvent system, acetic acid:dichloro-ethane:water (10:10:1, by vol., Gregg, 1966) for development. The proportions of the glycine and taurine conjugates of the major bile acids in bile were then determined by the $3 \alpha-$ (Bruusgaard, 1970) and 7 $\alpha$ - (Haslewood et al., 1973) hydroxysteroid dehydrogenase enzyme assays. The validity of this chromatographic-enzymatic technique

\footnotetext{
${ }^{1}$ Although the principal dihydroxy bile acids in bile are chenodeoxycholic acid ( $3 \alpha, 7 a$-dihydroxy-5 $\beta$-cholanic acid) and deoxycholic acid ( $3 \alpha-12 \alpha$-dihydroxy-5 $\beta$-cholanic acid) the analytical methods used in the present study would not allow the quantitative resolution of deoxycholic acid from the $7 \beta$ epimer of CDCA, ursodeoxycholic acid $(3 a, 7 \beta$-dihydroxy$5 \beta$-cholanic acid) which, according to Salen et al. (1974), Fromm et al. (1975) and Stiehl et al. (1975), may be found in the bile of patients during treatment with CDCA.
} 
was confirmed by comparing (1) the sum of the individual bile acid concentrations as assayed from the TLC plates with the total bile acid concentration as measured on non-chromatographed samples; and (2) the bile acid concentrations in the trihydroxy bile acid band when measured either with the $3 \alpha-$ or with the $7 \alpha$-enzymatic assays (both of which should measure identical concentrations of cholic acid).

\section{RELATIONSHIP BETWEEN BILE LIPIDS AND BILIARY BILE ACIDS}

To see if the saturation of bile with cholesterol was related to the proportion of CDCA in the biliary bile acids-or, indeed, to the proportion of the other major bile acids in bile - the saturation indices were plotted against the percentages of each of the major bile acids in turn. The statistical significance of the correlations between these variables was then calculated separately for patients starting and stopping treatment, and also when the data for the two groups were combined.

\section{Statistics}

The statistical significance of differences between the results was tested with Student's paired or unpaired two-tailed $t$ tests where appropriate.

\section{Results}

\section{BILE LIPID COMPOSITION}

\section{Patients starting treatment}

The results of bile lipid composition after starting treatment are shown for individual patients in Fig. 1, while the mean values ( \pm SEMs), grouped into specific time periods, are given in Fig. 2 (upper panel).

Before starting treatment the mean saturation index was $1.49 \pm 0.17$, the bile being supersaturated with cholesterol in five of the six patients, while in the remaining patient the SI of 0.96 was not significantly different from unity.

After starting oral CDCA, the mean time taken for bile to become unsaturated in cholesterol was $12 \pm$ 4.4 days but the pattern in individual patients was variable (Fig. 1). In three patients, for example, the saturation index fell to less than unity within the first week, while in others the bile remained supersaturated for up to three weeks. However, by four weeks, the bile had become undersaturated in all patients, by which time the bile lipid composition had become constant, both for individual patients (Fig. 1) and for the group as a whole (Fig. 2, upper panel).

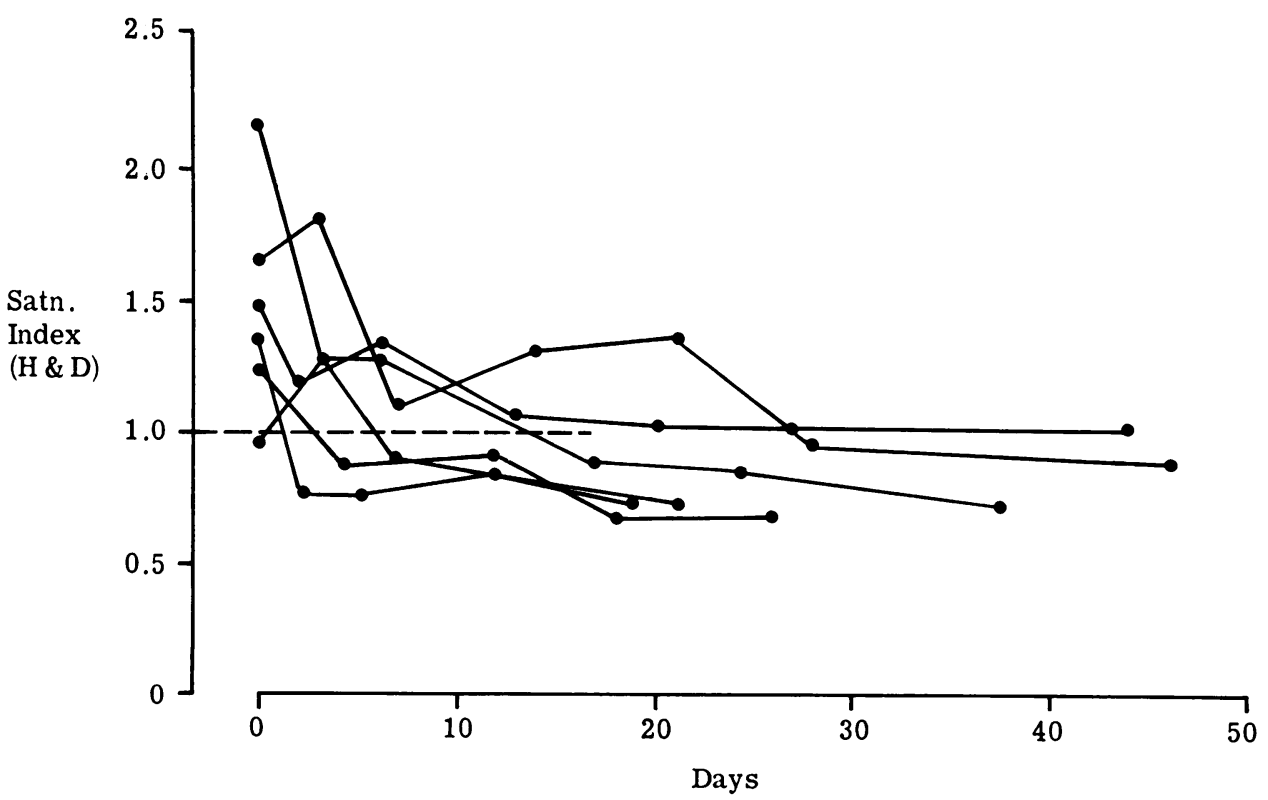

Fig. 1 Individual results of the biliary cholesterol saturation index (where 1.0 represents a saturated solution-criteria of Hegardt and Dam, 1971) in six gallstone patients before treatment (day 0) and at frequent intervals during the next 47 days after starting CDCA. 


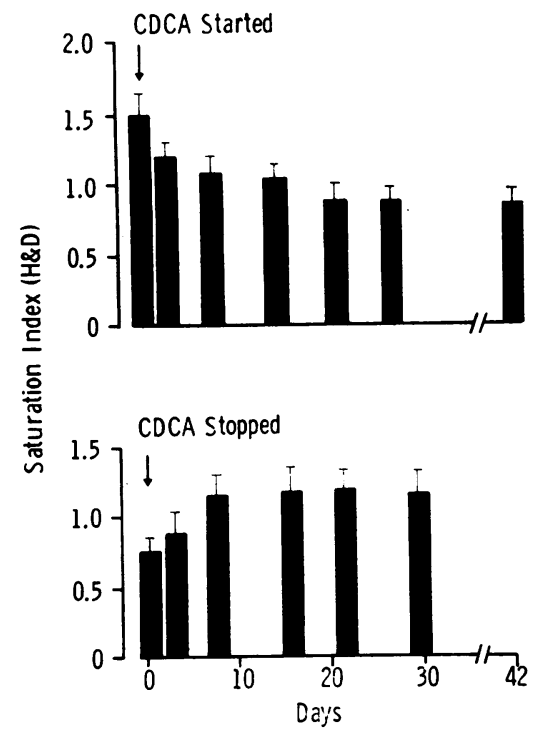

Fig. 2 Overall results of the biliary cholesterol SI (means $\pm S E M s$ ) in patients starting (upper panel: data from Fig. 1 grouped into different time periods) and stopping (lower panel) CDCA.

\section{Patients stopping treatment}

The mean biliary cholesterol saturation indices for patients stopping treatment are shown graphically in Fig. 2 (lower panel).

After withdrawing treatment, the mean saturation index increased rapidly from $0.74 \pm 0.10$ on day 0 , to $1 \cdot 15 \pm 0 \cdot 15$ by days $6-8$, and the mean time at which the bile became saturated ( $12 \pm 5.5$ days) was the same as that taken to produce unsaturated bile after starting CDCA. But, once again, there was a variable pattern of results in individual patients and by one week the bile had become supersaturated in

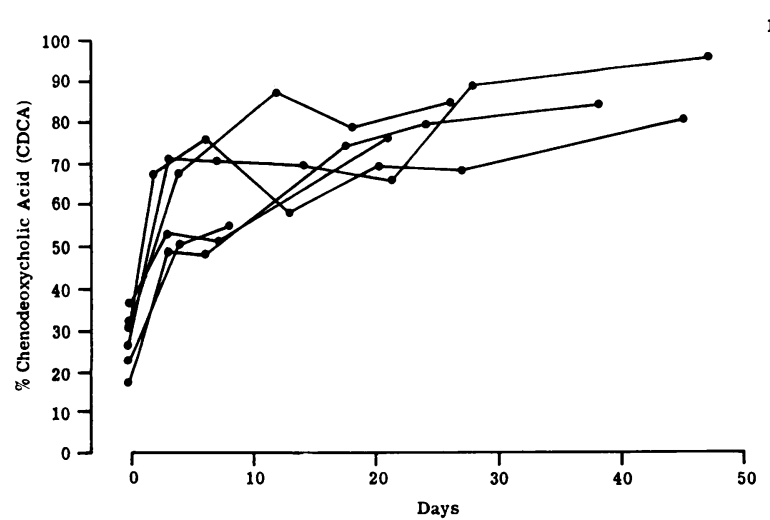

only three of the six patients. The rapid rise in the mean saturation index during the first week was largely due to values of 1.63 and 1.58 in two patients. In two other patients, the bile became supersaturated by the third week, while in the remaining patient, the bile did not revert to the supersaturated state until some time between day 20 and day 60 . Because of the wide scatter of results and the relatively small number of patients, it was not until days 20-22 that the mean saturation index of $1.20 \pm 0.16$ became significantly different from that on day $0(t=3 \cdot 153$; $\mathrm{P}<0.05$ ).

\section{BILIARY BILE ACIDS}

\section{Patients starting treatment}

Figure 3a shows the proportion of conjugated CDCA present in bile, expressed as a percentage of the total biliary bile acids in the individual patients. The percentage of conjugated CDCA increased rapidly in the first week from a mean of $27.9 \pm 2.5 \%$ on day 0 , to $60.5 \pm 4.2 \%$ on days $3-5(t=7.900 ; \mathrm{P}<0.001)$. Thereafter, the proportion of conjugated CDCA increased gradually to reach a mean of $80 \cdot 7 \pm 6 \cdot 2 \%$ during the fourth week.

The proportions of the other major bile acids present in bile are listed in Table 1. After starting CDCA, the percentage of the conjugates of the other primary bile acid, cholic acid, fell rapidly from $34.8 \pm$ $3 \cdot 1 \%$ to $12 \cdot 1 \pm 2 \cdot 0 \%$ by four days, and to $8 \cdot 3 \pm 1 \cdot 6 \%$ by eight days, after which it remained relatively constant. The proportion of deoxycholic acid conjugates also diminished but at a slower rate than did the proportion of conjugated cholic acid.

In spite of the increasing proportions of CDCA after starting treatment, there was no consistent change in the proportion of its bacterial metabolite, lithocholic acid. The percentage of conjugated

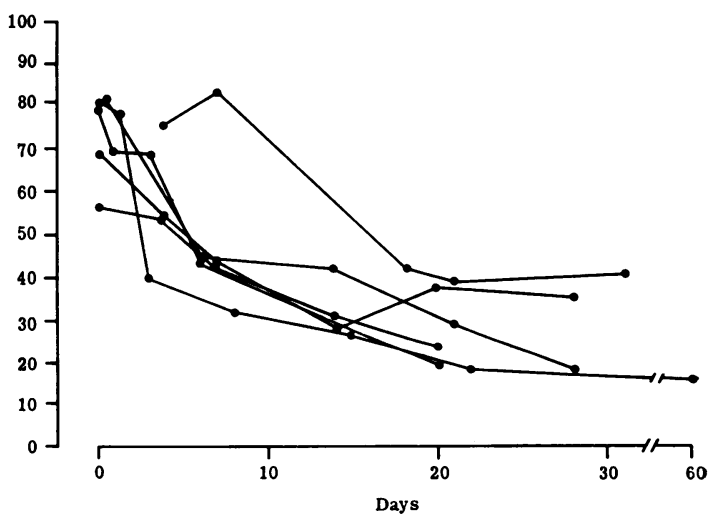

Fig. 3 The percentage of conjugated CDCA in biliary bile acids at different times after starting (a: left panel) and stopping (b: right panel) $C D C A$. 
Table 1 Biliary bile acid composition expressed as percentages (means $\pm S E M s$ ) before and at different times after starting $C D C A$

\begin{tabular}{|c|c|c|c|c|c|c|c|}
\hline \multirow[t]{2}{*}{ Bile acid } & \multirow[t]{2}{*}{ Before treatment } & \multicolumn{6}{|c|}{ Days after starting $C D C A$} \\
\hline & & $2-4$ & $6-8$ & $12-17$ & $18-24$ & $26-28$ & $38-47$ \\
\hline $\begin{array}{l}\text { Cholic } \\
\text { Deoxycholic } \\
\text { Lithocholic* }\end{array}$ & $\begin{array}{r}34.8 \pm 3.1 \\
34.8 \pm 1.6 \\
2.9 \pm 1.7\end{array}$ & $\begin{array}{r}12.1 \pm 2.0 \\
25.9 \pm 4.7 \\
1.8 \pm 0.7\end{array}$ & $\begin{array}{r}8.3 \pm 1.6 \\
30.6 \pm 6.2 \\
0.7 \pm 0.5\end{array}$ & $\begin{array}{r}8.9 \pm 1.5 \\
17.7 \pm 6.7 \\
1.6 \pm 0.9\end{array}$ & $\begin{array}{r}10.1 \pm 2.2 \\
12.6 \pm 3.6 \\
4.0 \pm 1.2\end{array}$ & $\begin{array}{r}7.2 \pm 0.6 \\
10.9 \pm 5.4 \\
1.7\end{array}$ & $\begin{array}{l}5 \cdot 1 \\
4 \cdot 6 \\
3 \cdot 9\end{array}$ \\
\hline
\end{tabular}

The values shown are for the glycine and taurine conjugates of the major bile acids in bile, with the exception of conjugated CDCA: these data are shown graphically in Fig. 3.

*'Lithocholic' refers to the conjugates of unsulphated lithocholic acid.

lithocholic acid varied considerably and the mean values during the first six weeks of treatment did not differ significantly from the results at day 0 , when the bile contained $2.9 \pm 1.7 \%$ of conjugated lithocholic acid. The maximum levels of conjugated lithocholic acid recorded were $8.7 \%$ in one patient before treatment and $8.6 \%$ in a different patient during CDCA therapy.

\section{Patients stopping treatment}

In patients whose gallstones had dissolved the proportion of conjugated CDCA fell rapidly in five of the six patients when treatment was withdrawn (Fig. 3b) from a mean of $73.7 \pm 4.9 \%$ on day 0 , to $34.3 \pm$ $3.2 \%$ at days $14-15$, at which time the mean percent of CDCA was not significantly different from the pretreatment level in patients starting therapy.

Table 2 lists the changes in the other major bile acids in patients stopping treatment. The proportion of conjugated cholic acid, which had fallen to $11.7 \pm$ $2.6 \%$ during treatment, returned to pretreatment levels by one to two weeks. Similarly, the diminished proportion of conjugated deoxycholic acid also returned to normal values when treatment was withdrawn, but, as in patients starting treatment, the changes in the proportion of this bile acid occurred more gradually than was seen for the conjugates of cholic acid.

Before stopping treatment, the levels of conjugated lithocholic acid in individual patients ranged from $0-4.6 \%$, and after an average of 19 months' treatment, the mean value of $1.6 \pm 0.9 \%$ was not sig- nificantly different from the mean pretreatment level. Again, the percent of conjugated lithocholate did not change significantly as CDCA was withdrawn.

\section{RELATIONSHIP BETWEEN BILIARY LIPIDS}

AND BILE ACIDS

As suggested by the results illustrated in Figs. 1 and 3 , there were statistically significant inverse relationships between the saturation indices and the percentages of conjugated CDCA in biliary bile acids, both for patients starting $(t=-4.671 ; \mathrm{P}<0.001)$ and stopping CDCA $(t=-3.097 ; \mathrm{P}<0.01)$. When the data for the two groups of patients were combined, there was a considerable scatter of results with a correlation coefficient of only 0.5 , but with 63 individual observations, the relationship between the two variables was, nonetheless, statistically significant $(t=-4.522 ; \mathrm{P}<0.001)$.

As a general rule, the bile became unsaturated with cholesterol when the proportion of CDCA exceeded $70 \%$. As can be seen in Fig. 4, although the bile occasionally became unsaturated before the proportion of CDCA reached $70 \%$, once this level had been passed, on only two out of 19 occasions did the bile remain supersaturated with cholesterol.

Although it seems likely that the saturation index is more dependent on the proportion of CDCA than on the proportion of the other bile acids in bile, there were, nonetheless, significant relationships between the saturation indices and the percentages of both cholic and deoxycholic acid. Again this was true, both for patients starting $(P<0.001$ for cholic acid;

Table 2 Biliary bile acid composition during (day 0) and at different times after stopping CDCA

\begin{tabular}{|c|c|c|c|c|c|c|}
\hline \multirow[t]{2}{*}{ Bile acid } & \multicolumn{6}{|c|}{ Days after stopping $C D C A$} \\
\hline & 0 & $3-4$ & $6-8$ & $14-18$ & $20-22$ & $28-31$ \\
\hline $\begin{array}{l}\text { Cholic } \\
\text { Deoxycholic } \\
\text { Lithocholic }\end{array}$ & $\begin{array}{r}11.7 \pm 2.6 \\
12.6 \pm 6.6 \\
1.6 \pm 0.9\end{array}$ & $\begin{array}{r}17.4 \pm 4.5 \\
21.8 \pm 2.6 \\
1.7 \pm 0.9\end{array}$ & $\begin{array}{r}29.6 \pm 6.0 \\
20.6 \pm 2.6 \\
1.5 \pm 0.3\end{array}$ & $\begin{array}{r}37 \cdot 2 \pm 2.6 \\
27.4 \pm 3.2 \\
1.5 \pm 0.2\end{array}$ & $\begin{array}{r}41.7 \pm 3.1 \\
29.1 \pm 4.6 \\
0.5 \pm 0.4\end{array}$ & $\begin{array}{r}33.9 \pm 4.5 \\
32.9 \pm 9.4 \\
1.6 \pm 0.9\end{array}$ \\
\hline
\end{tabular}




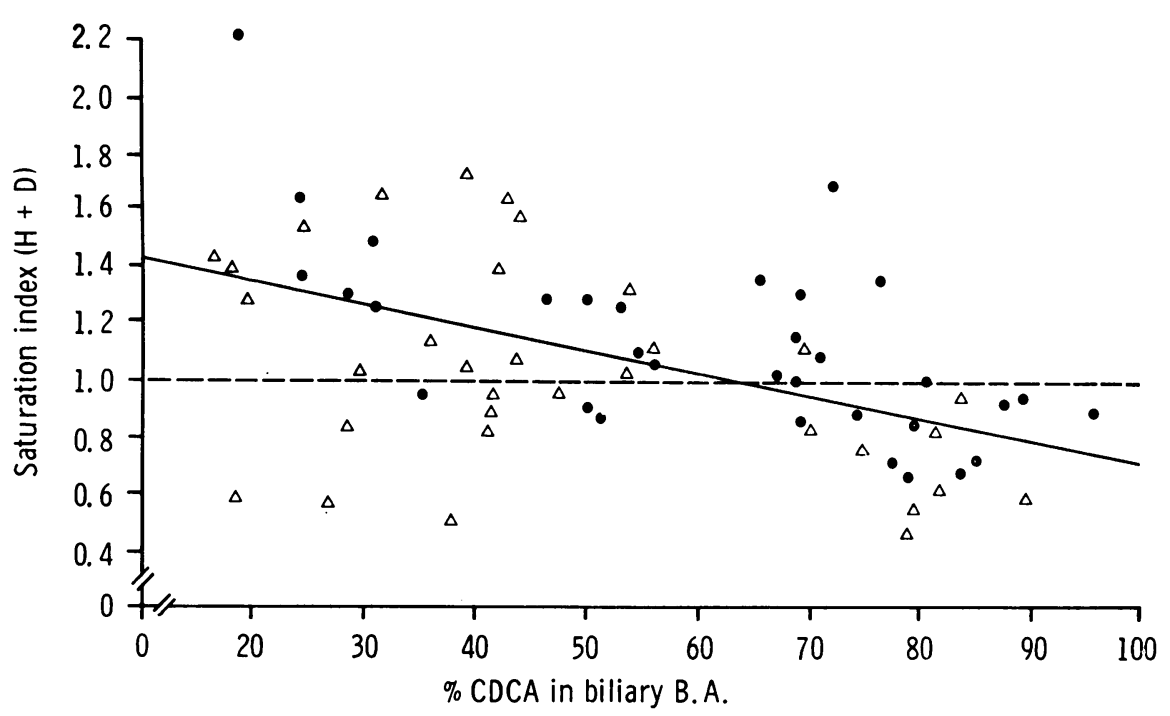

Fig. 4 Relationship between percent conjugated CDCA in the biliary bile acids and the cholesterol saturation index. The broken horizontal line represents a saturated solution (Hegardt and Dam, 1971), while the oblique line represents the regression line $y=1.62-0.009 x: n=63: r=-0.50: \mathrm{P}<0.001 .0=$ patients starting treatment with $C D C A$. $\Delta=$ patients stopping treatment.

$P<0.05$ for deoxycholic acid) and stopping CDCA $(P<0.02$ and $P<0.05$ respectively), and when the results for the two groups were combined $(P<0.001$ and $\mathrm{P}<0.002$ respectively).

\section{Discussion}

The results of these studies suggest that in patients with gallstones it takes from one to four weeks of CDCA therapy to produce an unsaturated bile, with the potential to dissolve gallstones, and that one should not look for representative changes in bile lipid composition in less than one month after starting treatment. The present findings also show that on stopping therapy, bile reverts to its supersaturated state in one to three weeks.

Before we could draw conclusions from these findings about the potential efficacy of intermittent CDCA treatment, we first had to compare the results from the two groups of patients, and, although they were not formally matched, they were, nonetheless, comparable with respect to age, sex, body weight, and the dose of CDCA prescribed, all of which are known to influence bile lipid composition (Dowling et al., 1972; Bennion and Grundy, 1975; Fisher and Yousef, 1976; Trash et al., 1976). Since the mean time taken for bile to become unsaturated was not shorter than that taken for bile to revert to its supersaturated state, it would seem that intermittent CDCA treatment would not effectively maintain a continuously unsaturated bile. Moreover, since gallstone dissolution both in vitro (Bell et al., 1972b) and in vivo (Iser et al., 1975) depends on the degree of desaturation of bile with cholesterol, it seems likely that intermittent treatment would only prolong the time required to dissolve the stones. Even with continuous therapy, the mean duration of treatment in the 18 patients from our own unit whose gallstones had completely dissolved, was $12.4 \pm 2.1$ months (range five to 38 ), so that any increase in the time taken to dissolve the stones would be undesirable.

The results in this paper were based on analyses of spot samples of bile-rich fluid aspirated from the duodenum after an overnight fast. We (Dowling $\boldsymbol{e t}$ al., 1972) and others (Thistle and Schoenfield, 1971; Vlahcevic et al., 1971) have previously shown that in patients undergoing cholecystectomy, the composition of gallbladder bile obtained at operation approximates closely to the composition of CCKstimulated bile-rich duodenal fluid aspirated before surgery. Furthermore, we have shown that bile lipid composition is relatively constant in individual patients and that there is close agreement between estimates of biliary cholesterol saturation indices measured on two or more occasions (Vlahcevic et al., 1971; Dowling et al., 1972). Prolonged secretion perfusion studies have also shown that the maximum saturation of bile with cholesterol occurs after an overnight fast (Northfield and Hofmann, 1975). An 
early morning spot sample, therefore, yields the most pessimistic estimate of the beneficial effects of CDCA on bile lipid composition.

There have been few previous studies either in animals or in man on the speed of change in bile lipid and bile acid composition after oral CDCA. Earlier, we had shown that after feeding $30 \mathrm{mg}$ CDCA.kg BW ${ }^{-1}$ day $^{-1}$ to the rhesus monkey, bile volume, bile acid, and bile lipid secretion changed promptly to reach a new steady state within 24-48 hours (Dowling, 1973), although the speed of change in bile acid secretion did seem to be dose dependent, taking 10 days to reach a steady state with a smaller dose $\left(5 \mathrm{mg} . \mathrm{kg} \mathrm{BW}^{-1} \mathrm{day}^{-1}\right)$. In the hamster, Goldstein et al. (1975) found that oral CDCA reduced the molar ratio of cholesterol in bile and that this reduction persisted for up to 20 days after the CDCA was withdrawn. In man, there has been only one previous study on the kinetics of change in bile lipids induced by CDCA. Marks et al. (1975) studied the effects of giving 125,250 , or $500 \mathrm{mg}$ CDCA/day to patients with gallstones before and at one and four weeks after starting therapy. The only patients in whom the bile became unsaturated were those given $500 \mathrm{mg} \mathrm{CDCA} /$ day for one month. Even then the bile was still saturated according to the limits defined by Hegardt and Dam (1971) and was unsaturated only by Admirand and Small's (1968) original criteriaa level which in our experience is inadequate to dissolve gallstones (Iser et al., 1975).

In the present study there was a considerable variation from patient to patient in the speed of change in bile lipid composition after starting and particularly after stopping CDCA treatment. The reason for this variation is unknown, but, theoretically, there are several factors which could have influenced the results in patients starting treatment. Apart from the dose of CDCA prescribed, a variable which we controlled within reasonably narrow limits, patient compliance in taking the medication, bioavailability and intestinal absorption of the CDCA capsules, and the biological variable of hepatic response to the ingested CDCA could all have affected the outcome. We have no reason to believe that the patients did not conscientiously take their capsules, at least during this short period of intensive study, and to date there is little information about the influence of the other factors on the bile lipid response to CDCA. Finally, the laboratory estimations of bile lipids could have modified the estimates of cholesterol saturation in bile, but given an accuracy of $\pm 5 \%$ in estimating bile acids, phospholipids, and cholesterol, we have calculated that, for bile samples with a saturation index of 1.0, the maximum variation due to the analytical technique would change the saturation index by not more than $\pm 0 \cdot 1$.

The long-term effects of CDCA feeding on biliary bile acid composition have been studied previously (Danzinger et al., 1973; Coyne et al., 1975; Fromm et al., 1975; Stiehl et al., 1975) and these investigators showed that during treatment the percent of CDCA conjugates reaches from $73-99 \%$. However, there have been no previous studies on the speed of change in biliary bile acids in patients starting and stopping CDCA therapy. As the percentage of CDCA conjugates increases in patients starting treatment, the reciprocal fall in the proportion of cholic acid conjugates is not unexpected since it is known that oral CDCA depresses both cholate synthesis and pool size (Danzinger et al., 1973). In turn, since cholate is the precursor of its bacterial metabolite deoxycholate, it is not surprising that the proportion of this secondary bile acid should also fall progressively in patients starting treatment.

It should be noted that the $7 \alpha$-hydroxysteroid dehydrogenase enzyme assay used in the present study would not detect the presence of the $7 \beta$ epimer of chenodeoxycholate, ursodeoxycholic acid, which also runs in the dihydroxy bile acid TLC band (Haslewood and Haslewood, 1974). (Indeed it has recently been implied that during 'chenotherapy', the CDCA may be partly converted to ursodeoxycholic acid and that the beneficial effects of CDCA on cholesterol solubility in bile may actually be mediated by its conversion to ursodeoxycholate (Carey, 1975).) After four weeks' treatment, however, and in patients whose gallstones had just been dissolved, the sum of the cholate, CDCA, and lithocholate levels meant that there was less than $13 \%$ 'deoxycholate' present in the biliary bile acids. It is unlikely, therefore, that significant amounts of ursodeoxycholate had been formed.

Just as the amount of deoxycholic acid present in bile is linked to the amount of its precursor, cholic acid, so the proportion of lithocholate in bile might be expected to reflect the increasing percentages of CDCA in patients starting treatment and the decreasing percentages of 'cheno' in the bile of patients whose treatment was withdrawn. It is fortunate that this was not the case, since lithocholate is potentially hepatotoxic (Palmer, 1976) and the absence of lithocholate accumulation in the present studies is in accord with results from other units ( $\mathrm{La}$ Russo et al., 1975; Bremmelgaard and Pedersen, 1976). This failure to accumulate lithocholate during 'chenotherapy' is probably due to the high rate of lithocholate sulphation by the human liver (Palmer and Bolt, 1971; Cowen et al., 1975a) and the poor reabsorption of lithocholate sulphates from the intestine (Low-Beer et al., 1969; Cowen et al., 1975b). Thus, the formation of bile acid sulphates represents 
an efficient safety mechanism in man. Since the bile samples were not solvolysed in the present study, the proportions of sulphated bile acids were not measured.

The correlation between the saturation index and the percentage of CDCA in biliary bile acids showed that in patients with gallstones, when the bile contained more than $70 \%$ CDCA it was almost invariably undersaturated with cholesterol and, while this suggests that the increased proportion of CDCA was responsible for the improvement in cholesterol solubility in bile, the present results can tell us nothing about the mechanisms for the change in bile lipid composition. Indeed, our results show that the saturation index also correlates significantly with the percentages of cholate and deoxycholate and since deoxycholate feeding actually increases the saturation of bile with cholesterol, Low-Beer and Pomare (1975) have postulated that the proportion of deoxycholate has an important role in controlling cholesterol solubility in bile.

Although the results in this paper suggest that intermittent CDCA will not continuously maintain bile in an unsaturated state, long-term intermittent CDCA could still be effective in reducing or preventing gallstone recurrence once the stones have been dissolved. This hypothesis must await the proof of clinical experience, but in our own series there have been six gallstone recurrences in five patients out of a total of 17 patients whose gallstones had been completely dissolved and who have been followed up for a mean period of 12 months. Our present policy is to stop CDCA treatment once two consecutive cholecystograms, three months apart, have confirmed complete gallstone dissolution. Until we know the natural history of the frequency and timing of gallstone recurrence after CDCA withdrawal, we cannot logically test whether long-term low or full dose maintenance treatment, intermittent CDCA therapy or perhaps dietary measures (Pomare et al., 1974) will be necessary.

We thank Miss Elizabeth Sabin for technical assistance, and Charge Nurse John Howes for help with the intubation procedures. We are grateful to Weddel Pharmaceuticals Ltd, for supplies of chenodeoxycholic acid (Chendol) and financial support: J.H.I. is a Weddel Research Fellow. We are indebted to Mrs Hazel Creed for secretarial assistance.

\section{References}

Adler, R. D., Bennion, L. J., Duane, W. C., and Grundy, S. M. (1975). Effects of low dose chenodeoxycholic acid feeding on biliary lipid metabolism. Gastroenterology, 68, 326334.

Admirand, W. H., and Small, D. M. (1968). The physico- chemical basis of cholesterol gallstone formation in man. Journal of Clinical Investigation, 47, 1043-1052.

Bell, G. D., Whitney, B., and Dowling, R. H. (1972a). Gallstone dissolution in man using chenodeoxycholic acid. Lancet, 2, 1213-1216.

Bell, G. D., Sutor, D. J., Whitney, B., and Dowling, R. H. (1972b). Factors influencing human gallstone dissolution in monkey, dog and human bile. Gut, 13, 836. (Abstract).

Bennion, L. J., and Grundy, S. M. (1975). Effects of obesity and caloric intake in biliary lipid metabolism in man. Journal of Clinical Investigation, 56, 996-1011.

Bremmelgaard, A., and Pedersen, L. (1976). Bile acids in bile during long-term chenodeoxycholic acid treatment. Scandinavian Journal of Gastroenterology, 11, 161-165.

Bruusgaard, A. (1970). Quantitative determination of the major 3-hydroxy bile acids in biological material after thinlayer chromatographic separation. Clinica Chimica Acta, 28, 495-504.

Carey, M. C. (1975). Cheno and urso-what the goose and the bear have in common. New England Journal of Medicine, 293, 1255-1257. (Editorial).

Coyne, M. J., Bonorris, G. G., Chung, A., Goldstein, L. I., Lahana, D., and Schoenfield, L. J. (1975). Treatment of gallstones with chenodeoxycholic acid and phenobarbital. New England Journal of Medicine, 292, 604-607.

Cowen, A. E., Korman, M. G., Hofmann, A. F., and Cass, O. W. (1975a). Metabolism of lithocholate in healthy man. I. Biotransformation and biliary excretion of intravenously administered lithocholate, lithocholylglycine, and their sulfates. Gastroenterology, 69, 59-66.

Cowen, A. E., Korman, M. G., Hofmann, A. F., Cass, O. W., and Coffin, S. B. (1975b). Metabolism of lithocholate in healthy man. II. Enterohepatic circulation. Gastroenterology, 69, 67-76.

Danzinger, R. G. Hofmann, A. F., Schoenfield, L. J., and Thistle, J. L. (1972). Dissolution of cholesterol gallstones by chenodeoxycholic acid. New England Journal of Medicine, 286, $1-8$.

Danzinger, R. G., Hofmann, A. F., Thistle, J. L., and Schoenfield, L. J. (1973). Effect of oral chenodeoxycholic acid on bile acid kinetics and biliary lipid composition in women with cholelithiasis. Journal of Clinical Investigation, 52, 2809-2821.

Dowling, R. H. (1973). Ninth Symposium on Advanced Medicine, p. 379. Edited by G. Walker. Pitman Medical: London.

Dowling, R. H., Bell, G. D., and White, J. (1972). Lithogenic bile in patients with ileal dysfunction. Gut, 13, 415-420.

Fisher, M. M., Price, V. M., and Yousef, I. M. (1976). Biliary lipids in pregnancy. In The Hepatobiliary System, p. 555. Edited by W. Taylor. Plenum: New York. (In press).

Fromm, H., Holz-Slomczyk, M., Zobl, H., Schmidt, E., and Schmidt, F. W. (1975). Absence of hepatotoxicity in patients treated with chenodeoxycholic acid (CDC) for gallstones. Gastruenterology, 69, 822. (Abstract).

Goldstein, L. I., Bonorris, G. G., Coyne, M. J., and Schoenfield, L. J. (1975). Persistent effects of chenodeoxycholic acid on biliary lipids in the hamster. Journal of Laboratory and Clinical Medicine, 85, 1032-1041.

Gregg, J. A. (1966). New solvent systems for thin-layer chromatography of bile acids. Journal of Lipid Research, 7, 579-581.

Halsewood, G. A. D., Murphy, G. M., and Richardson, J. M. (1973). A direct enzymatic assay for $7 \alpha$-hydroxy bile acids and their conjugates. Clinical Science, 44, 95-98.

Haslewood, E. S., and Haslewood, G. A. D. (1974). Specificity and some characteristics of a $7 a$-hydroxysteroid dehydrogenase from $E$. coli. In Advances in Bile Acid Research, p. 105 . Edited by S. Matern, J. Hackenschmidt. P. Back, and W. Gerok Schattauer: Stuttgart. 
Hegardt, F. G., and Dam, H. (1971). The solubility of cholesterol in aqueous solutions of bile salts and lecithin. Zeitschrift für Ernährungswissenschaft, 10, 223-233.

Hofmann, A. F., and Paumgartner, G. (eds) (1975). Chenodeoxycholic Acid Therapy of Gallstones. Schattauer: Stuttgart.

Iser, J. H., Dowling, R. H., Mok, H. Y. I., and Bell, G. D. (1975). Chenodeoxycholic acid treatment of gallstones: A follow-up report and analysis of factors influencing response to therapy. New England Journal of Medicine, 293, 378-383.

La Russo, N. F., Hoffman, N. E., Hofmann, A. F., Northfield, T. C., and Thistle, J. L. (1975). Effect of primary bile acid ingestion on bile acid metabolism and biliary lipid secretion in gallstone patients. Gastroenterology, 69, 13011314.

Low-Beer, T. S., and Pomare, E. W. (1975). Can colonic bacterial metabolites predispose to cholesterol gallstones? British Medical Journal, 1, 438-440.

Low-Beer, T. S., Tyor, M. P., and Lack, L. (1969). Effects of sulfation of taurolithocholic and glycolithocholic acids on their intestinal transport. Gastroenterology, 56,721-726.

Marks, J., Bonorris, G., Chung, A., Coyne, M., Goldstein, L., Okun, R., and Schoenfield, L. (1975). Feasibility of low dose and intermittent chenodeoxycholic acid therapy of gallstones. Gastroenterology, 68, 946. (Abstract).

Mok, H. Y. I., Bell, G. D., and Dowling, R. H. (1974a). Effect of different doses of chenodeoxycholic acid on bilelipid composition and on frequency of side-effects in patients with gallstones. Lancet, 2, 253-257.

Mok, H. Y. I., Perry, P. M., and Dowling, R. H. (1974b). The control of bile acid pool size: effect of jejunal resection and phenobarbitone on bile acid metabolism in the rat. Gut, 15, 247-253.

Northfield, T. C., and Hofmann, A. F. (1975). Biliary lipid output during three meals and an overnight fast. I. Relationship to bile acid pool size and cholesterol saturation of bile in gallstone and control subjects. Gut, 16, 1-11.

Northfield, T. C., La Russo, N. F., Hofmann, A. F., and Thistle, J. L. (1975). Biliary lipid output during three meals and an overnight fast. II. Effect of chenodeoxycholic acid treatment in gallstone subjects. Gut, 16, 12-17.

Palmer, R. H. (1976). Toxic effects of lithocholate on the liver and biliary tree. In The Hepatobiliary System, p. 227. Edited by W. Taylor. Plenum: New York. (In press).

Palmer, R. H., and Bolt, M. G. (1971). Bile acid sulfates. I. Synthesis of lithocholic acid sulfates and their identification in human bile. Journal of Lipid Research, 12, 671-679.

Pomare, E. W., Heaton, K. W., Low-Beer, T. S., and White, C. (1974). Effect of wheat bran on bile salt metabolism and bile composition. Gut, 15, 824-825. (Abstract).

Salen, G., Tint, G. S., Eliav, B., Deering, N., and Mosbach, E. H. (1974). Increased formation of ursodeoxycholic acid in patients treated with chenodeoxycholic acid. Journal of Clinical Investigation, 53, 612-621.

Stiehl, A., Raedsch, R., and Kommerell, B. (1975). Increased sulfation of lithocholate in patients with cholesterol gallstones during chenodeoxycholate treatment. Digestion, 12, 105-110.

Thistle, J. L., and Schoenfield, L. J. (1971). Induced alterations in composition of bile of persons having cholelithiasis. Gastroenterology, 61, 488-496.

Thistle, J. L., Yu, P. Y. S., Hofmann, A. F., and Ott, B. J. (1974). Prompt return of bile to supersaturated state followed by gallstone recurrence after discontinuance of chenodeoxycholic acid therapy. Gastroenterology, 66, 789. (Abstract).

Thomas, P. J., and Hofmann, A. F. (1973). A simple calculation of the lithogenic index of bile: expressing biliary lipid composition on rectangular co-ordinates. Gastroenterology, 65, 698-700 (Letter).

Trash, D. B., Ross, P. E., Murison, J., and Bouchier, I. A. D. (1976). The influence of age on cholesterol saturation of bile. Gut, 17, 394. (Abstract).

Vlahcevic, Z. R., Bell, C. C., Juttijudata, P., and Swell, L. (1971). Bile-rich duodenal fluid as an indicator of biliary lipid composition and its applicability to detection of lithogenic bile. American Journal of Digestive Diseases, 16, 797-802. 